\title{
Vacuum Ultraviolet and Visible Spectroscopy Diagnostics on the NOVA-UNICAMP Tokamak
}

\author{
A.M. Daltrini, M. Machida, and M.J.R. Monteiro \\ Instituto de Física "Gleb Wataghin", \\ Universidade Estadual de Campinas, \\ C. P. 6165, 13083-970, Campinas, SP, Brazil
}

Received on 1 March, 2001

\begin{abstract}
Three visible and one VUV spectrometers covering four toroidal positions have been set to study $\mathrm{He}$ and $\mathrm{H}$ plasma created by NOVA-UNICAMP tokamak. Ion temperatures have been measured at the beginning of the tokamak discharge by Doppler broadening of $\mathrm{C}, \mathrm{O}$ and He lines. The time evolution of carbon line emissions, with different degrees of ionization, showed to have opposite behavior between high and low plasma ionization condition. The high density operation and 2 $\mathrm{kHz}$ oscillation present throughout discharge, caused by periodical touching of the plasma with NOVA-UNICAMP tokamak limiter, restrained the increase of ion temperature.
\end{abstract}

\section{Introduction}

Light emission from the hydrogen and helium plasmas, as well as impurity emissions, have been studied in the visible and vacuum ultraviolet (VUV) spectra, by a set of four spectrometers installed around toroidal equatorial plane of NOVA-UNICAMP tokamak. The use of VUV spectroscopy to study tokamak confined plasmas for the first time in domestic level also is pointed out.

The spectroscopy is a powerful diagnostic tool to study magnetically confined plasma, providing important parameters as ion temperature and density, $Z_{\text {eff }}$, and particle confinement time. The VUV spectroscopy becomes very important at high temperatures, since the emissions of the mostly ionized species is concentrated in VUV and X-ray spectra.

Doppler broadening of line spectra using the wellknown formula [1,2] determines the ion temperature

$$
T=1.69 \times 10^{8} M\left(\frac{\Delta \lambda_{D}}{\lambda_{0}}\right)^{2}
$$

where, the ion temperature $T$ is in $\mathrm{eV}, \Delta \lambda_{D}$ (full width at half height) centered at the wavelength of the observed spectra $\lambda_{0}$, and $M$ is the ion mass in atomic units.

Actual value of instrumental broadening effect is taken into account by the formula $[1,3]$

$$
\left(\Delta \lambda_{\text {meas }}\right)^{2}=\left(\Delta \lambda_{D}\right)^{2}+\left(\Delta \lambda_{\text {ins }}\right)^{2}
$$

where instrumental broadening $\left(\Delta \lambda_{\text {ins }}\right)$ is assumed to have a gaussian shape, fact verified by measurements, and Stark or Zeeman $[3,4]$ effects are considered to be negligible $[1,2]$.

Therefore the measurement of a spectral line broadening and the utilization of formulas (1) and (2), allow us to obtain the ion temperature $\mathrm{T}$ using CIII and OII emissions in the visible region and CIV in the VUV region.

The four spectrometers, see Fig. 1, set around equatorial plane of tokamak are: a 82-415 Jarrel-Ash Spectrometer with $25 \mathrm{~cm}$ focal distance, $590 \mathrm{~g} / \mathrm{mm}$ and 66 $\AA / \mathrm{mm}$ dispersion is set at the limiter position $\left(0^{0}\right)$, a 2051 McPherson Spectrometer with $100 \mathrm{~cm}$ focal distance, $1200 \mathrm{~g} / \mathrm{mm}$ and $8,3 \AA / \mathrm{mm}$ dispersion is set at $90^{\circ} \mathrm{cw}$ from the limiter position, a SPEX Spectrometer with $75 \mathrm{~cm}$ focal distance, $1200 \mathrm{~g} / \mathrm{mm}$ and $11 \AA / \mathrm{mm}$ dispersion is set at $180^{\circ}$, and a $225 \mathrm{McPherson}$ Spectrometer with $100 \mathrm{~cm}$ focal distance, $600 \mathrm{~g} / \mathrm{mm}$ and $16,6 \AA / \mathrm{mm}$ dispersion is set at $225^{\circ}$. The last measures radiation in VUV and visible up to $6000 \AA$ and has a differential pumping, with a turbomolecular pump, in the pipeline which connect spectrometer to the tokamak.

Formerly, in measurements using hydrogen gas lamp, the VUV spectrometer measured radiation down to $500 \AA$ [5]. However, measuring radiation emitted by our tokamak plasma, the lowest wavelength detectable was $900 \AA$, probably because of weak line signals in the tokamak plasma and grating deterioration.

For the visible spectrometers, $\Delta \lambda_{\text {ins }}$ have been determined using a He-Ne laser. However, since the VUV spectrometer can not measure the He-Ne laser wavelength, we use for this propose a carbon line emission 
in the VUV region (1548 $\AA$ ) using discharges with very low values of ohmic heating and magnetic fields. In this case, by the formula (1), the Doppler broadening is very small, and total width measured can be considered as the instrumental broadening [1].

Finally, we also present the time behavior of carbon lines with different degrees of ionization, using discharges with low ohmic heating and magnetic fields. Although, in this mode, we can not measure $\Delta \lambda_{D}$, the intensity measurements allow us to drive some conclusions.

The line intensity of a given ionized specie is proportional to its density, and is given by:

$\frac{d N_{j}}{d t}=-n_{e} I_{j} N_{j}-n_{e} \alpha_{j} N_{j}+n_{e} I_{j-1} N_{j-1}+n_{e} \alpha_{j+1} N_{j+1}+\phi_{j}$

where $N_{j}$ is the density of the ion ( $j$ represents the ionization level), $n_{e}$ is the electron density, $I_{j}$ is the ionization rate coefficient, $\alpha_{j}$ is the recombination rate coefficient and $\phi_{j}$ is the source term.

A expression for the $I_{j}$ coefficients is present by Kunze [6]:

$$
I_{j} \cong 7.5 \times 10^{-8} \frac{\eta_{j}}{E_{j}}\left[\left(\ln \frac{40 T_{e}}{E_{j}}\right)^{3}+40\right]
$$

$$
\times \frac{T_{e}^{1 / 2}}{E_{j}+3 T_{e}} e^{-E_{j} / T_{e}} \mathrm{~cm}^{3} / \mathrm{s}
$$

where $\eta_{j}$ is the number of electrons in the $j$ th shell, $E_{j}$ is the ionization energy and $T_{e}$ is the electron temperature (both $E_{j}$ and $T_{e}$ are in $\mathrm{eV}$ ).

Equations (3) and (4) and line intensity measurements are used to study the fast heating and posterior cooling of the plasma related to the ionization levels of carbon lines.

\section{Experimental Setup}

The NOVA-UNICAMP tokamak, former NOVA II tokamak [7] from Kyoto University-Japan, is a small machine operating at our Laboratory since 1996. Its main characteristics are: major radius of $30 \mathrm{~cm}$, minor radius of $6 \mathrm{~cm}$, plasma current of $10 \mathrm{kA}$, plasma discharge time of $15 \mathrm{~ms}$, and toroidal magnetic field of $1 \mathrm{~T}$.

A schematic view of the NOVA-UNICAMP tokamak and main diagnostics installed, microwave interferometer, ruby laser Thomson scattering, hard x-ray detector, partial pressure gauge and the spectrometers, can be seen in Fig.1. In Fig. 2 is shown a typical plasma current and loop voltage signals.

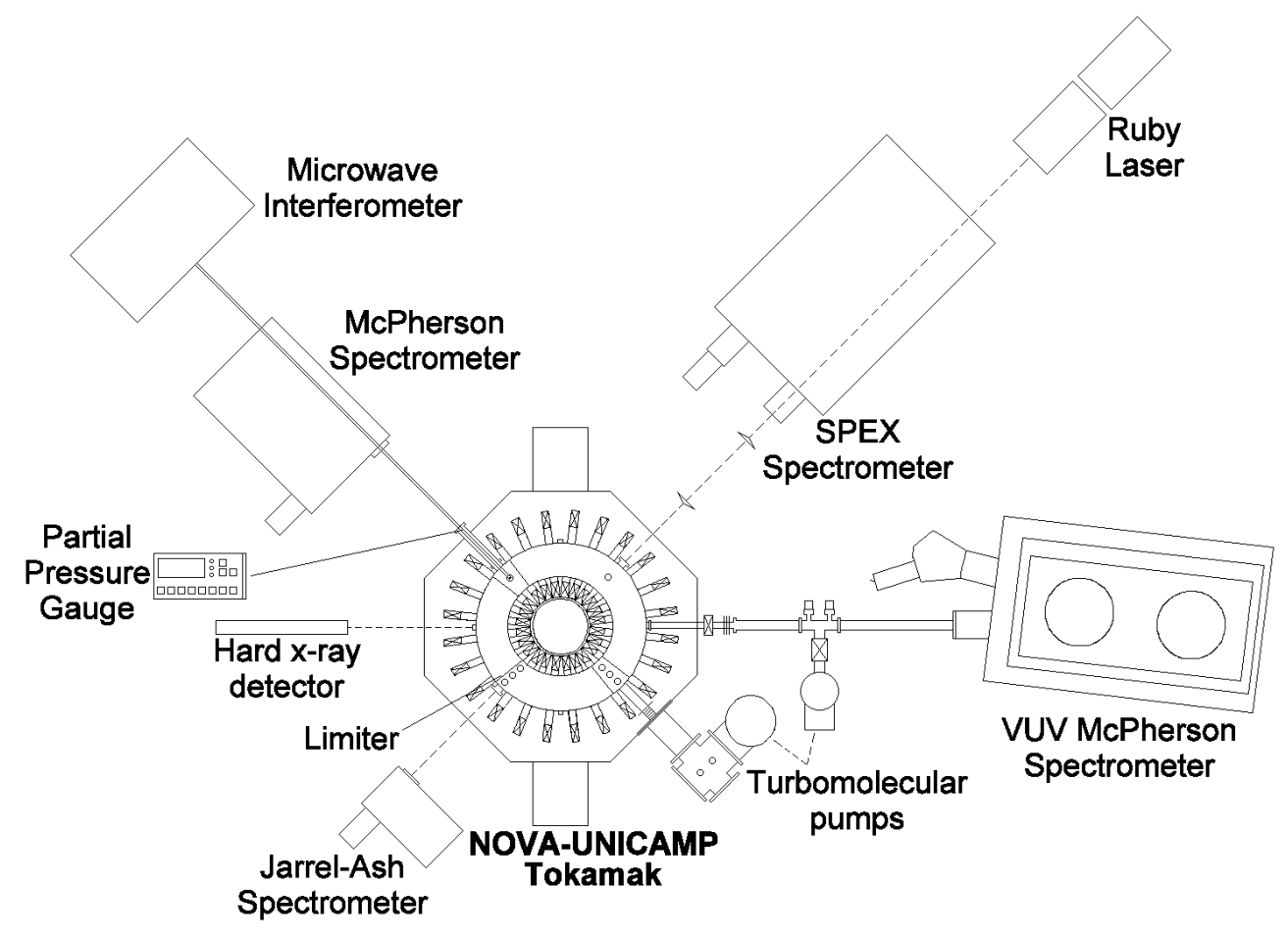

Figure 1. NOVA-UNICAMP tokamak and its main diagnostics. 


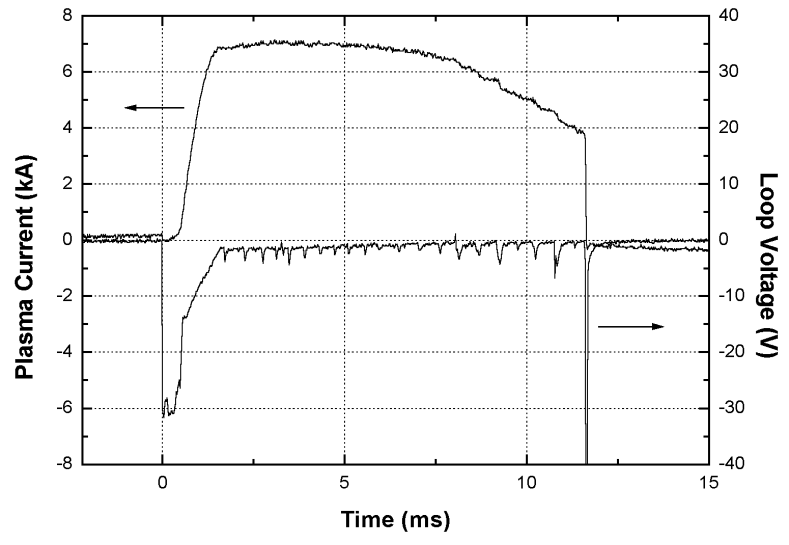

Figure 2. Typical plasma current and loop voltage signals in $\mathrm{H}$ gas discharges.

\section{Experimental Results}

The ion temperature has been determined by Doppler broadening line measurements. Using simultaneously four spectrometers we could cover the VUV and visible regions and therefore measuring the most convenient spectral lines for each ion.

The spectral lines used for ion temperature calculations were: HeI $6678 \AA$ in helium plasma, OII $4649 \AA$, CIII $4647 \AA$ and CIV $1548 \AA$ for hydrogen plasma. The temperatures were calculated nearly up to the middle of each discharge. After this point, because of unknown noise effects in the signals, the temperature evolution was not determined.

Usually for ion temperature determination, one should choose well isolated spectra line. In Fig. 3 (top) is shown CIII and OII lines in visible region, where, due to overlapping effect, temperature determinations are jeopardized. Different Doppler broadening and temperatures, $35 \pm 14 \mathrm{eV}$ and $23.9 \pm 5.2 \mathrm{eV}$, are obtained for each peak.

At same figure (bottom), where CIV line in VUV region is taken, the spectra is much well defined. The temperature $63.2 \pm 9.4 \mathrm{eV}$ is basically the same for two peaks, as should be. The higher temperature is indication that the CIV emission comes from hotter plasma region.

Other CIV emissions in visible region also have been investigated, presenting very weak signals however, and showing again the importance of the VUV spectroscopy.
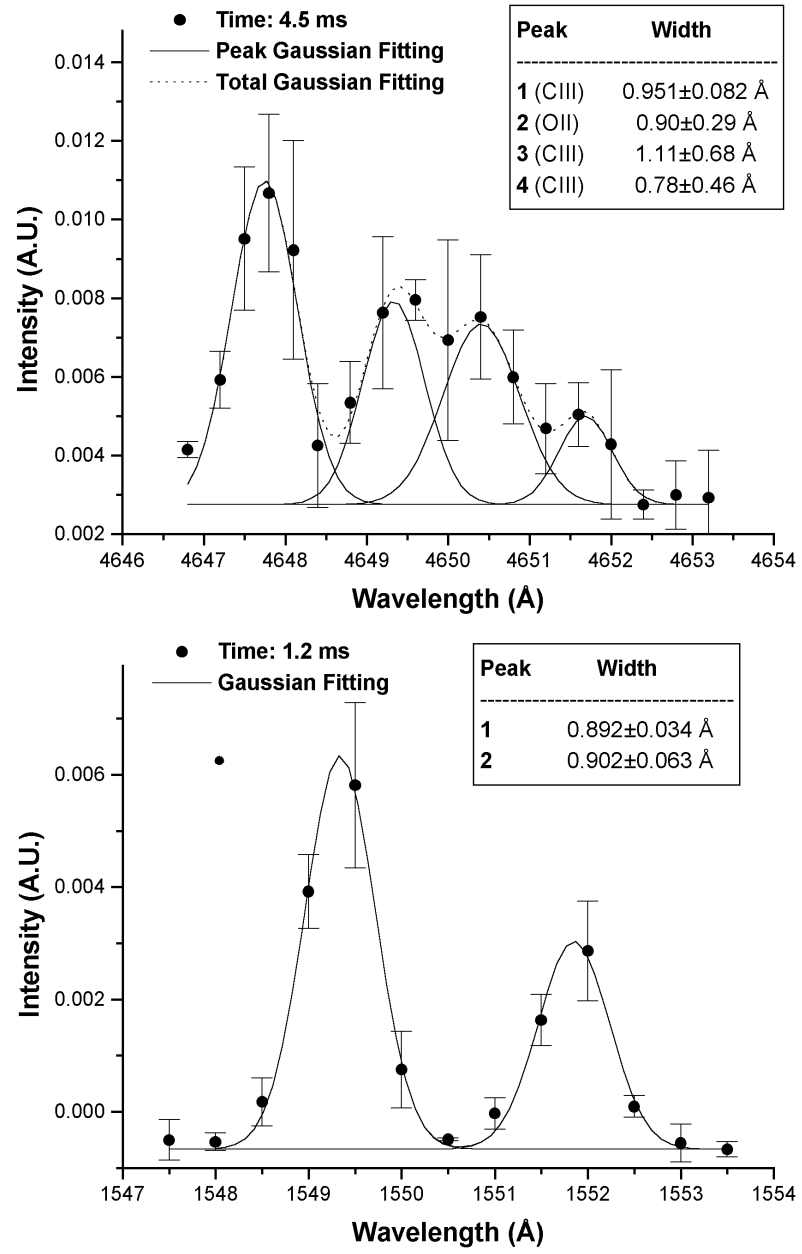

Figure 3. Top: Overlapping of CIII and OII four emission spectra in visible region; notice Doppler broadening deviation for each spectra giving different temperatures for same species. Bottom: VUV spectra of CIV emission; notice very well defined Doppler broadening for both emissions.

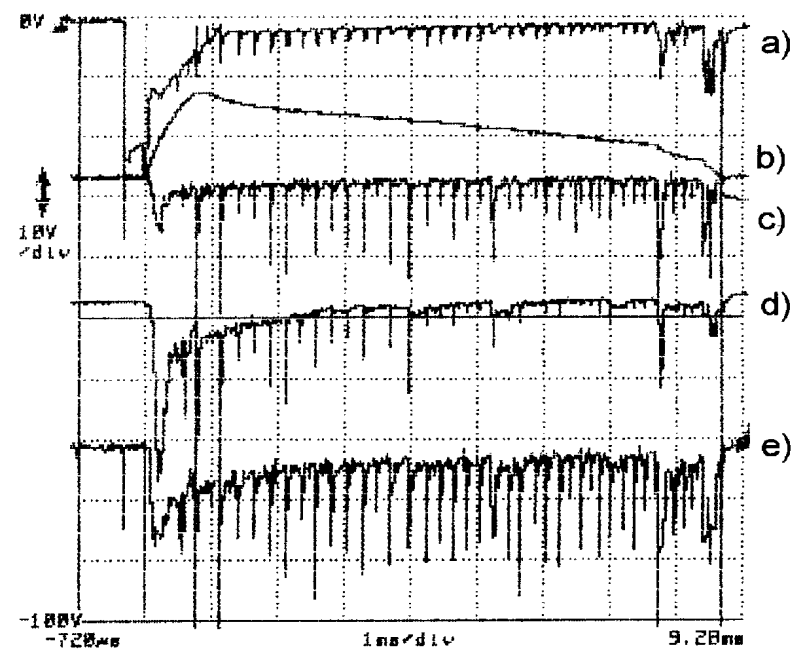

Figure 4. Oscillations of $2 \mathrm{kHz}$ peaks, in loop voltage and spectral line signals at three toroidal positions: a) Loop Voltage; b) Plasma Current; c) $H_{\alpha}-0^{0}$; d) $H_{\alpha}-90^{0}$; e) $H_{\alpha^{-}}$ $180^{\circ}$.

However, temperature values are lower than that 
we expected for this machine [7]. Probably, this fact is related with the oscillations observed in loop voltage and spectral line signals. In Fig. 4, the simultaneous measurements of the loop voltage, plasma current, and $H_{\alpha}$ emissions in three different toroidal positions are shown. We observed that oscillations of about $2 \mathrm{kHz}$ occur at the same time in all toroidal positions during the discharge, but they do not have the same intensity variations. The VUV spectrometer at $225^{\circ}$, measuring $H_{\beta}$ emission, also presented similar results.

These oscillations is seen to be caused by periodical touching of the plasma with limiter, creating high density, $\sim 3 \times 10^{13} \mathrm{~cm}^{-3}$, operation of the NOVAUNICAMP tokamak.

The gas recycling from wall is very common problem in small machines like our tokamak. Fast neutrals produced by charge exchange leave the confinement, reaching the wall, and are trapped, originating the quick decay of plasma density. This will cause sliding away of plasma and touching the limiter, increasing the density again.

In the case of helium operation, as can be seen at Fig. 5, the periodical touching of plasma with the limiter is avoided. This is because the gas recycling is done properly, since the helium neutrals are not stick to the wall as hydrogen, producing therefore more constant plasma density and stable confinement.

The comparison between helium and hydrogen plasma discharges show that the He plasma reaches stable condition faster than the $\mathrm{H}$ plasma. As can be seen from Fig. 6, He temperature reach the mean value just a few moments, $0.5 \mathrm{~ms}$, after the beginning of the discharge. However, it does not happen with hydrogen plasma, showing higher temperature only after $4 \mathrm{~ms}$.

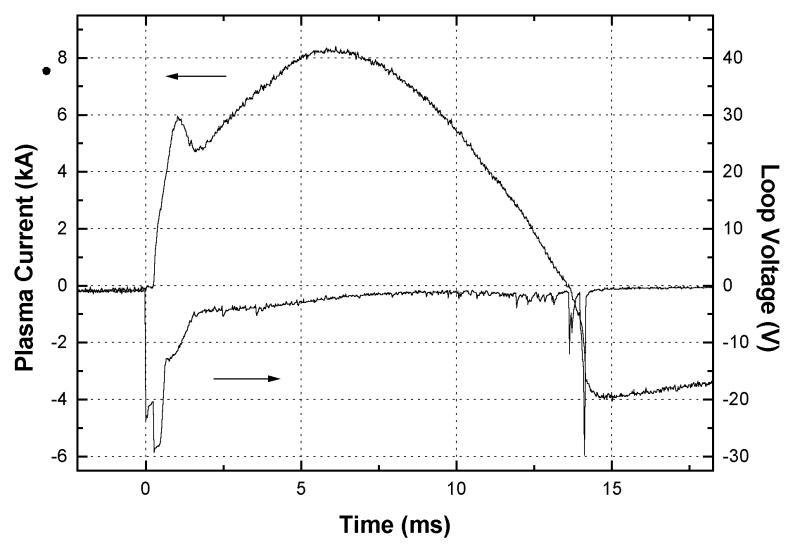

Figure 5. Plasma current and loop voltage signals in helium discharge. Observe the absence of oscillations.

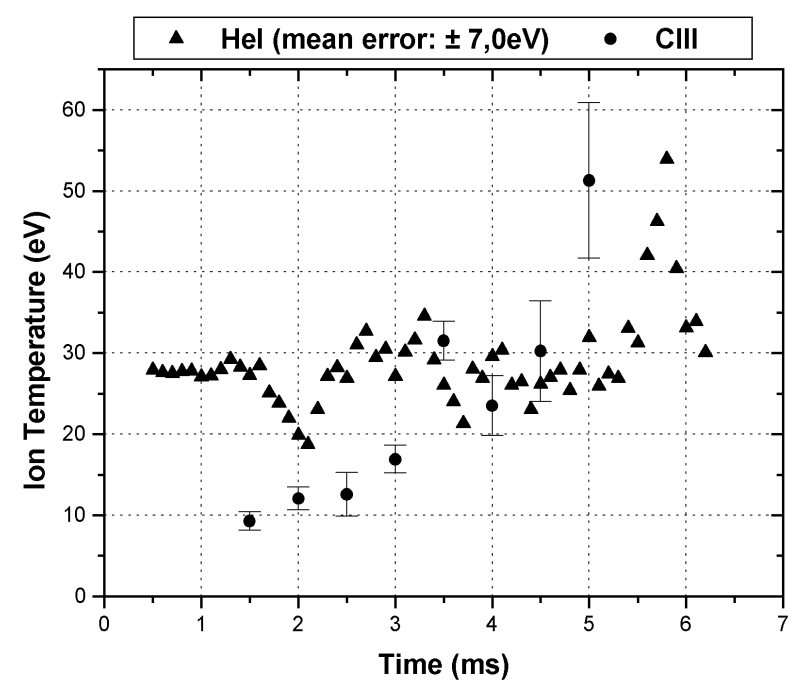

Figure 6. HeI (helium plasma) and CIII (hydrogen plasma) ion temperature.

Using the VUV McPherson spectrometer, the time behavior of carbon lines with different ionization degrees have been studied. We have used discharges with low ohmic heating and magnetic field (toroidal magnetic field $=0,3-0,4 \mathrm{~T}$ ). In this mode we obtain discharges with high degree of reproducibility, in order to provide more accurate comparisons.

The carbon lines CI $2967 \AA$, CII $4267 \AA$, CIII 2297 $\AA$ and CIV $1548 \AA$, together with the typical hydrogen plasma current and loop voltage signals, are shown in Fig. 7. The spectral line signals are taken in different, but similar discharges at the same toroidal position.

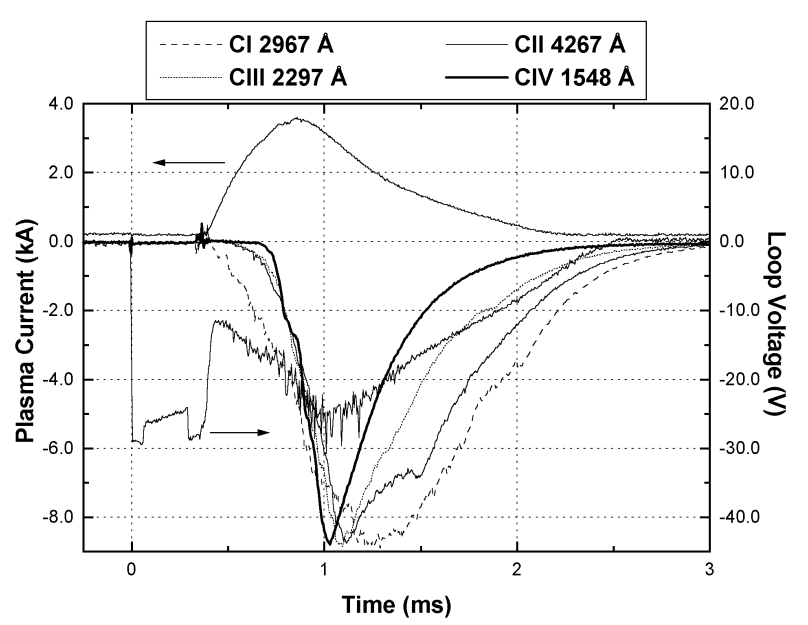

Figure 7. Different ionized carbon emissions.

In Fig. 8, a zoom of the carbon line signals are presented, and where we can observe that the appearance of CI line starts earlier and lasts for longer time, whereas the rise and fall time of the highly ionized CIV line is much faster, reaching the peak value first. 
The line start delay is explained by the fact that the plasma takes a certain time to heat and then, to produce more ionized ions. In this way, as the plasma current is established, we observe first CI emission. After, the temperature starts to increase and we see the beginning of the CII emission, followed by the CIII and CIV (more hot plasma) emissions.

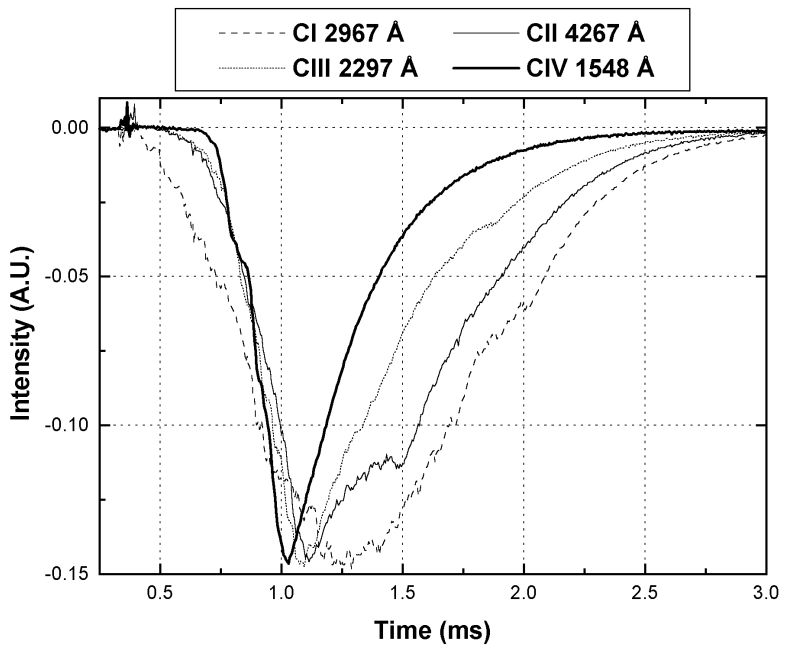

Figure 8. Different ionized carbon emissions (zoom).

The order of appearance of line peaks as observed in the Fig. 8 is opposed to what is usually observed in tokamaks [8] and other fusion machines [9]. The difference here is that the plasma is not totally ionized.

Even in bigger tokamaks, the electron temperature is not so high in the first moments of the discharge [8]. For example, for $3 \mathrm{eV}$ electrons, eq. (4) gives $I_{0} \approx 3 \times 10^{-9} \mathrm{~cm}^{3} / \mathrm{s}$ and $I_{2} \approx 5 \times 10^{-16} \mathrm{~cm}^{3} / \mathrm{s}$. Once in our machine the density of this mode is approximately $10^{12} \mathrm{~cm}^{-3}$, we have $n_{e} I_{0} \approx 3 / \mathrm{ms}$ and $n_{e} I_{2} \approx 5 \times 10^{-7} / \mathrm{ms}$.

Since the time between the plasma current beginning and the CIV peak is less than $1 \mathrm{~ms}$, we confirm, observing these values and the eq. (3) that the plasma is not totally ionized.

The low plasma duration is caused by low magnetic mode discharge, so that the plasma is not well confined. As the plasma column is formed, it expands and touches the limiter (maximum plasma current). After that, the plasma becomes cooler, and the most ionized ions, which are present in low quantities, have populations (and emissions) reduced very fast.

In the case of a long duration discharge with a plasma totally ionized, the particles are heat progressively, so that the number of low ionized species will be reduced with time, leading to opposed results that observed here.

A similar effect of temperature decrease soon after the plasma current peak, can also be seen in the measurements of the HeI temperature in low field discharges. Due to very low ion temperature operation, we have used HeI line instead of impurity lines, as carbon, once Doppler broadening is very hard to be separated from the instrumental broadening. In the Fig. 9, we can see that the ion temperature is increasing approximately up to the plasma current peak (time zero) and then decreases until reach values undetectable by our measurements.

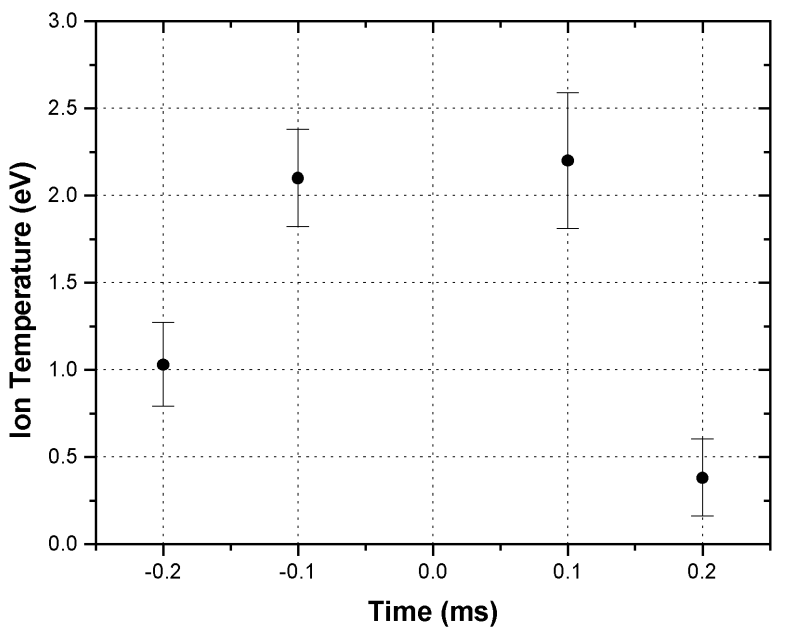

Figure 9. HeI temperature in low energy discharge.

\section{Conclusions}

The VUV spectroscopy showed to be a powerful diagnostic combined with visible spectroscopy. Simultaneous spectral measurements at the four tokamak toroidal positions showed that the $2 \mathrm{kHz}$ oscillations are global effect having different intensities. For the low density and weak magnetic field discharges, where full ionization is not reached during the short lived plasma, the appearance order of the CI, CII, CIII and CIV lines are opposite to the usual tokamak or theta-pinch discharges with fully ionized plasma. The ion temperature measurements in tokamak condition showed that helium plasma reaches constant temperature much faster than looking at CIII impurity line in the hydrogen discharge.

\section{Acknowledgements}

We would like to acknowledge Prof. Dr. Masayuki Fukao for his efforts to bring NOVA tokamak to our laboratory and for his precious and continuos help in this research.

This work is supported by FAPESP - Fundação de Amparo à Pesquisa do Estado de São Paulo, FINEP, CNPq and CAPES. 


\section{References}

[1] A.M. Daltrini, M. Sc. Thesis (IFGW-UNICAMP 08/1999).

[2] R.H. Huddlestone and S. L. Leonard, Plasma Diagnostic Techniques, Academic Press (1965).

[3] I. Condrea, E. Haddad, B.C. Gregory, D. Lafrance, J.L. Lachambre, G. Pacher, F. Meo and H.H. Mai, Rev. Sci. Instrum. 70, 387 (1999).

[4] D.P. Stotler, C.H. Skinner and C.F.F. Karney, Rev. Sci. Instrum. 70, 347 (1999).
[5] S.A. Moshkalyov, M. Machida, S.V. Lebedev and D.O. Campos, Revist. de Fís. Aplic. e Instr. 10, 107 (1995).

[6] H. -J. Kunze, Phys. Rev. A 3, 937 (1971).

[7] M. Fukao, K. Mori and T. Tanihara, Plasma Phys. Control. Fusion 33, 199 (1991).

[8] Equipe TFTR, Nucl. Fusion 15, 1053 (1975).

[9] M. Machida, S.V. Lebedev, S.A. Moshkalyov, D.O. Campos and L.A. Berni, Braz. J. Phys. 26, 747 (1996). 